\title{
The NRG I exon I I missense variant is not associated with autism in the Central Valley of Costa Rica
}

\author{
Lynne A McInnes*1,2, Leonid Ouchanov ${ }^{1}$, Alisa Nakamine, \\ Patricia Jimenez ${ }^{3}$, Marcela Esquivel ${ }^{3}$, Marietha Fallas ${ }^{3}$, Silvia Monge ${ }^{3}$, \\ Pamela Bondy ${ }^{4}$ and Elina R Manghi ${ }^{4}$
}

Address: ${ }^{1}$ Department of Psychiatry, Mount Sinai School of Medicine, New York, New York, USA, ${ }^{2}$ Department of Human Genetics, Mount Sinai School of Medicine, New York, New York, USA, ${ }^{3}$ Hospital Nacional de Ninos "Dr Sáenz Herrera"; CCSS, Child Developmental and Behavioral Unit, San José, Costa Rica and ${ }^{4}$ University of Illinois at Chicago, Chicago, Illinois, USA

Email: Lynne A McInnes* - alison.mcinnes@mssm.edu; Leonid Ouchanov - leonid.ouchanov@mssm.edu;

Alisa Nakamine - alisa.nakamine@mssm.edu; Patricia Jimenez - draprjg@yahoo.com; Marcela Esquivel - esquivel_marcela@yahoo.com;

Marietha Fallas - draprjg@yahoo.com; Silvia Monge - smongeq@rasca.co.cr; Pamela Bondy - Pbondy@uic.edu;

Elina R Manghi - elina25@uic.edu

* Corresponding author

Published: 22 May 2007

BMC Psychiatry 2007, 7:21 doi:10.1 |86/|47|-244X-7-21
Received: 23 November 2006

Accepted: 22 May 2007

This article is available from: http://www.biomedcentral.com/I47I-244X/7/2I

(c) 2007 Mclnnes et al; licensee BioMed Central Ltd.

This is an Open Access article distributed under the terms of the Creative Commons Attribution License (http://creativecommons.org/licenses/by/2.0), which permits unrestricted use, distribution, and reproduction in any medium, provided the original work is properly cited.

\begin{abstract}
Background: We are conducting a genetic study of autism in the isolated population of the Central Valley of Costa Rica (CVCR). A novel Neuregulin I (NRGI) missense variant (exon II $\mathrm{G}>\mathrm{T}$ ) was recently associated with psychosis and schizophrenia (SCZ) in the same population isolate.
\end{abstract}

Methods: We genotyped the NRGI exon II missense variant in I 46 cases with autism, or autism spectrum disorder, with CVCR ancestry, and both parents when available $(\mathrm{N}=267$ parents) from 143 independent families. Additional microsatellites were genotyped to examine haplotypes bearing the exon II variant.

Results: The NRGI exon I/ G>T variant was found in 4/I46 cases including one de novo occurrence. The frequency of the variant in case chromosomes was 0.014 and 0.045 in the parental non-transmitted chromosomes. At least 6 haplotypes extending $0.229 \mathrm{Mb}$ were associated with the $\mathrm{T}$ allele. Three independent individuals, with no personal or family history of psychiatric disorder, shared at least a I megabase haplotype 5 ' to the T allele.

Conclusion: The NRGI exon II missense variant is not associated with autism in the CVCR.

\section{Background}

We are conducting a population genetic study of autism in the CVCR [1]. Recently, Walss-Bass et al. [2] reported that they had identified a novel missense variant in the transmembrane domain of Neuregulin 1 (Val>Leu in exon 11) associated with the phenotypes of psychosis (SCZ and psychotic mood disorder) and SCZ in the CVCR. The cases apparently descended from a common ancestor who migrated to Costa Rica from Spain and was born in 1670 . Genome-wide linkage studies of autism have not highlighted chromosome 8p12, where NRG1 is located, and autism is not characterized by psychosis. However, both 
autism and SCZ have been linked to abnormal expression of the Reelin (RELN) gene (reviewed in Fatemi 2005 [3]). Additionally, deletions of $22 \mathrm{q} 11.2$ are associated both with autism and psychosis $[4,5]$ and another group demonstrated that offspring of parents with psychosis or affective disorders had a greater risk of developing autism [6]. Finally, SCZ is widely present in the relatives of our autism probands and these probands have been ascertained from the CVCR as in the Walss-Bass study. Given these data suggesting a common genetic basis for at least a subset of autism and SCZ subjects, and the shared ancestry of the autism and SCZ probands in both studies, we decided to screen our cases for the presence of the NRG1 exon 11 $\mathrm{G}>\mathrm{T}$ missense variant.

\section{Methods \\ Subjects}

We are collecting autism trios with the goal of performing a population genetic study of autism in the isolated founder population of the CVCR. As this population was founded by a small number of individuals in the $16^{\text {th }}$ century, and grew exponentially in isolation until the 1970s, disease alleles present at the founding should be widely distributed in the current population. This project was approved under the guidelines of the Ministry of Health of Costa Rica, the Ethical committee of the National Children's Hospital in San Jose (Hospital Nacional de Ninos or HNN), and the Institutional Review Board at Mount Sinai School of Medicine in accordance with the Declaration of Helsinki. These approvals remain active.

Details of the case ascertainment and evaluation process have been previously described [1]. Briefly, families of individuals with possible autism contact us, or are contacted by us after expressing interest in the study, and are formally asked to participate using established informed consent criteria. All interviews and exams take place in the Neurodevelopmental Unit of the HNN. Possibly affected individuals and their parents are interviewed by experienced neuropediatricians using the Autism Diagnostic Interview Revised (ADI-R) [7]. The Autism Diagnostic Observation Schedule (ADOS) [8] is also administered to subjects and both of these assessments are videotaped for independent scoring by the best estimators. IQ tests are administered that are appropriate for the age and level of verbal communication of the subjects. The Vineland Adaptive Behavioral Scales [9] are administered and compared with the results of IQ tests to confirm diagnoses of mental retardation.

All subjects are evaluated with a complete medical and neurological examination, including a dermatological examination with Wood's Lamp to look for signs of tuberous sclerosis and Hypomelanosis of Ito. All subjects in this study have been reported negative for FRAXA muta- tions and subjects with mental retardation were reported to have normal G-banded karyotypes at 550-band resolution.

Subjects were included in this study if they met DSM IVTR [10] criteria for autism in all three symptom domains (social interaction, communication or language, and behavioural abnormalities) as measured by the ADI-R and the ADOS. Additionally, age of onset for at least one symptom domain must have been less than 36 months. A diagnosis of autism spectrum was given to individuals who met ADI-R criteria but had an age of onset greater than 36 months, or were no more than one point below ADI-R criteria for autism in the social domain, and in either the communication or repetitive behaviour domains, but not in both, or if they met full criteria using the ADI-R but were one point below the cut-off score for autism in one domain of the ADOS. We also included subjects with a DSM IV-TR diagnosis of Asperger's Syndrome. Although we will not include probands with a DSM IV-TR diagnosis of Pervasive Developmental Disorder Not Otherwise Specified (PDD-NOS) in a future whole genome association study of autism, we did include them in this exploratory study. Subjects with a nonverbal IQ $<35$ were excluded unless they had an adaptive behaviour score much higher than the IQ measure. This is because there are no local Costa Rican norms for IQ testing and thus IQ may be underestimated in this population [1].

All individuals also had to have CVCR ancestry defined as having at least $6 / 8$ great-grandparents (GGPs) born in the CVCR, or all 4 GGPs from either the maternal or paternal side (in which case only transmission from the parent with CVCR ancestry is considered). Furthermore, individuals cannot be related by fewer than 6 generations, although we did genotype siblings, if affected, in the current study. The sample genotyped for this study includes 143 independent families. There were 129 cases with autism, 67\% of which also had a diagnosis of mental retardation. The rest of the cases did not have mental retardation and included $\mathrm{N}=7$ with autism spectrum disorder, $\mathrm{N}=4$ with PDD-NOS, and $\mathrm{N}=3$ with Asperger's Syndrome. Three autism cases also had siblings with either PDD-NOS (2), or autism (1). The mean age of the subjects was 5.75 years and the range was $3-13$. Sixteen of the 143 cases were female. The three multiplex families consisted of two male sib pairs and one male:female sib pair.

\section{Genotyping}

A TaqMan SNP genotyping assay was designed using ABI Assay-by-Design File Builder Version 2.0 from the following genomic sequence: 
GAACATGGACAATGTCATGCAGCATGCCCACTGTTTGG TTGTAGTCAGTCCTGGCAAGTGGAAGTGACCTGTGATG ACATCTGCTCTCATCCCTTTCCAGAGGCGGAGGAGCT GTACCAGAAGAGAGTGCTGACCATAACCGGCATCTGC ATCGCCCTCCTTGTGGTCGGCATCATGTGT [G/ T]TGGTGGCCTACTGCAAAACCAAGTAAACCTTCTTTCT CCATGCСTTTCTCTCTCCTTCATGCAGAGACAGCTTAG ATGGCCAGGGCTTTGCAGAATCTGAGCTCCACAGCCTAGTCTTGGGG.

The sequences used for the amplification primers are as follows: Forward primer, TGCATCGCCCTCCTTGTG; Reverse primer, AGAAAGGCATGGAGAAAGAAGGTTT.

Identification of the $\mathrm{G}$ allele was accomplished using the probe CACCACACACATGAT-8 labelled with VIC. Identification of the $\mathrm{T}$ allele was performed using CCACCAAACACATGAT-8 labelled with FAM. The SNP Genotyping Assay Mix was obtained from Applied Biosystems (Foster City, CA) at a concentration of $40 \times$, and diluted to $20 \times$ with $1 \times$ TE. For each reaction, $2.50 \mu \mathrm{l}$ of $2 \times$ TaqMan Universal PCR Master Mix, No AmpErase UNG (Applied Biosystems, Foster City, CA), 0.25 ul of $20 \times$ SNP Genotyping Assay Mix, and $2.25 \mu \mathrm{l}$ of genomic DNA at 10 $\mathrm{ng} / \mu \mathrm{l}$ were used for a total reaction volume of $5 \mu \mathrm{l}$ using 384-well plates. The plates were run in the ABI 7900 thermal cycler with the following conditions: $95^{\circ} \mathrm{C}$ for 10 minutes hold, then 40 cycles of 15 second denaturation at $92^{\circ} \mathrm{C}$ and 1 minute of annealing/extension at $60^{\circ} \mathrm{C}$. At the completion of the amplification and the absolute quantification read, the allelic discrimination assay read was performed to generate the allelic discrimination read file to be analyzed on SDS 2.1.

Microsatellite markers for haplotype analysis included 317J8-2123, D8S1810, 420M9-1395 and 487-2. Details regarding genotyping of these microsatellites are available upon request. Note that the order of these markers has changed in the Human Genome Browser March 2006 assembly since the publication of NRG1 haplotypes by Li et al. [10]. D8S1810 and 420M9-1395 have switched positions. Details regarding the microsatellite markers we utilized are available at deCODE Genetics [14].

\section{Results}

Frequency of the exon II missense variant in cases and parental non-transmitted chromosomes

The exon 11 missense variant was present in two cases diagnosed with autism, one case diagnosed with an autism spectrum disorder (one point below one domain of the ADI-R), and one case diagnosed with PDD NOS, in addition to co-morbid attention deficit hyperactivity disorder and a tongue tic (Case 129). The allele was transmitted from the mother in all cases but appears to have occurred de novo in Case 129. The missense allele was also present in 12 unrelated, other than by marriage, parental individuals, 7 fathers and 5 mothers, from 11 families who did not transmit the allele to cases. To be clear, both parents of Case 25 carry the T allele and they are not related to each other by fewer than 6 generations. The frequency of the allele in the non-transmitted chromosomes was therefore $12 / 267=0.045$, remarkably close to the frequency estimate of 0.042 by Walss-Bass et al [2]. Haplotype analysis revealed the presence of 6 haplotypes carrying the missense $\mathrm{T}$ allele when considering only the closest microsatellite $317 \mathrm{~J} 8-2123,0.229 \mathrm{Mb}$ proximal to the exon 11 missense variant (Table 1). Please see Figure 1 for the position of microsatellite markers typed in relation to the exon 11 variant. After microsatellite 317J82123, the next closest markers are clustered together with D8S1810 at $\sim 0.964 \mathrm{Mb}$ upstream followed by 420M91395 and then 487-2 1.04 Mb upstream from the first marker. These three 5 ' markers were typed as most associations to NRG1 with SCZ have been to markers in this region. Three parental chromosomes from 3 independent families (one transmitted to a case and the other two nontransmitted) shared alleles at all 4 microsatellite markers typed over a distance of $\sim 1.04 \mathrm{Mb}$ (Table 1 ). Two other independent parental non-transmitted chromosomes also may share a different $\sim 1.04 \mathrm{Mb}$ haplotype however phase is uncertain.

\section{Psychiatric history of exon II missense variant carriers}

We take a careful family psychiatric history on first, second and third degree relatives of all probands. Therefore, we reviewed the psychiatric history of all cases as well as persons carrying the $\mathrm{T}$ allele to look for the presence of psychotic or affective disorders. None of the 12 'T' carrier non-transmitting parents had a psychiatric disorder. However, AU025C, a 40 year old mother did have a sister with manic-depression and, AU113C, a 38 year old mother, did have a brother who had been hospitalized with SCZ (unfortunately DNA samples could not be obtained on these subjects). This inheritance pattern could represent incomplete penetrance in carriers of the missense allele. However, 113C and 25C did not share a haplotype with each other. Of note, 7 trios not segregating the T allele had a blood-related aunt or uncle with schizophrenia, which were not available for testing. An $8^{\text {th }}$ case had a parent

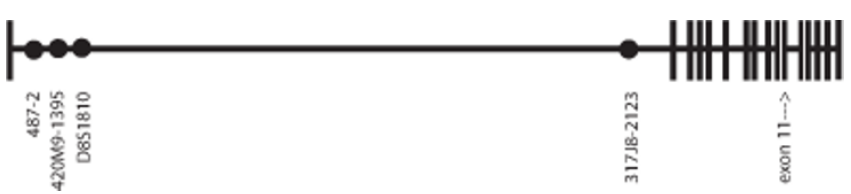

Figure I

Position of the microsatellite markers genotyped in NRG I. Diagram not to scale with regards to the position of exons. 
Table I: Microsatellite haplotypes observed in exon I I missense variant carriers.

\begin{tabular}{|c|c|c|c|c|c|c|c|c|c|}
\hline Family ID & $\mathrm{A} / \mathrm{B} / \mathrm{C} / \mathrm{D}$ & $487-2$ & D8S 1810 & $420 M 9-1395$ & $317 J 8-2123$ & Exon $\| \mathrm{G}>\mathrm{T}$ & $\mathrm{T} / \mathrm{NT}$ & Hap\# & Comment \\
\hline AU025 & $25 B$ & 247.5 & 194 & 190 & 240 & $\mathrm{~T}$ & NT & I & \\
\hline AU025 & $25 \mathrm{C}$ & 235.7 & 192 & 202 & 247 & $\mathrm{~T}$ & NT & 2 & Sister with bipolar disorder \\
\hline AU027 & $27 C$ & 247.5 & 194 & $(190)$ & 246 & $T$ & NT & 3 & Possible extended hap \\
\hline AU044 & $44 C$ & 247.5 & $(194)$ & 190 & 246 & $T$ & $N T$ & 3 & Possible extended hap \\
\hline AU058 & 58A & 245.7 & 176 & 181 & 247 & $\mathrm{~T}$ & $\mathrm{~T}$ & 2 & \\
\hline AU058 & $58 C$ & 245.7 & 176 & $|8|$ & 247 & $\mathrm{~T}$ & $\mathrm{~T}$ & 2 & \\
\hline AU058 & $58 \mathrm{D} 2$ & 245.7 & 176 & $|8|$ & 247 & $\mathrm{~T}$ & $\mathrm{~T}$ & 2 & \\
\hline AU059 & 59A & 235.7 & 196 & 202 & 242 & $\mathrm{~T}$ & $\mathrm{~T}$ & 4 & \\
\hline AU059 & $59 C$ & 235.7 & 196 & 202 & 242 & $\mathrm{~T}$ & $\mathrm{~T}$ & 4 & \\
\hline AU06I & $61 \mathrm{~B}$ & 237.7 & 178 & 181 & 242 & $T$ & NT & 4 & Extended hap \\
\hline AU07I & $7 I B$ & 237.7 & 178 & $|8|$ & 242 & $T$ & NT & 4 & Extended hap \\
\hline AU090 & 90A & 237.7 & 178 & $|8|$ & 242 & $T$ & $\mathrm{~T}$ & 4 & Extended hap \\
\hline AU090 & $90 \mathrm{C}$ & 237.7 & 178 & 181 & 242 & $T$ & $\mathrm{~T}$ & 4 & \\
\hline AU089 & $89 C$ & 246.4 & 176 & 181 & 242 & $T$ & NT & 4 & Extended hap w recomb? \\
\hline AUII3 & $113 \mathrm{C}$ & 253.1 & 178 & 186 & 242 & $\mathrm{~T}$ & NT & 4 & Brother with SCZ \\
\hline$A \cup 129$ & I29A & 235.7 & 194 & 202 & 246 & $\mathrm{~T}$ & NT & 3 & De novo in case \\
\hline AUI43 & I43B & 243.8 & 194 & 202 & 253 & $\mathrm{~T}$ & NT & 5 & \\
\hline AUI64 & $164 \mathrm{~B}$ & 241.8 & 178 & 186 & 253 & $\mathrm{~T}$ & NT & 5 & \\
\hline AUI65 & $165 B$ & 239.7 & 196 & 190 & 238 & $\mathrm{~T}$ & NT & 6 & \\
\hline
\end{tabular}

The first column contains the family ID number of the autism proband. The second column indicates the family member status, for example $25 \mathrm{~A}$ signifies the case, 25B signifies the father, 25C signifies the mother and 58D2 indicates the second sibling of case 58. The next 4 columns contain alleles from the 4 microsatellites genotyped to determine which haplotypes are associated with the rare ' $T$ ' allele. Alleles were binned where possible with the exception of marker 478-642 wherein the allele is called as reported by the software. Brackets around an allele mean that phase could not be determined with certainty. The "T/NT" column indicates whether the haplotype was transmitted, or not transmitted, from a parent to a case, or the sibling of a case. The "Hap\#" column indicates the number of different haplotype backgrounds associated with the ' $T$ ' allele considering only the closest microsatellite 3I7J8-2I 23 (0.229 Mb away). The comment column notes extended haplotypes > I Mb A question mark next to the haplotype number indicates that the haplotype background could not be determined with certainty.

with SCZ who did not carry the T allele and a parental sib with SCZ whose carrier status is unknown. A $9^{\text {th }}$ autism case had more than one first degree relative with SCZ, neither of whom carried the $\mathrm{T}$ allele. None of the three $\mathrm{T}$ allele transmitting parents had a personal or family history of psychotic or affective disorders. Finally, Case 129 appears to carry a de novo occurrence of the T allele as we have observed no other non-mendelian transmissions from either parent to Case 129 in a panel of markers spanning chromosomes 15, 7, 17, 21 and 4 which should effectively rule out non-paternity.

\section{Discussion}

Our major conclusion is that the NRG1 missense variant is not associated with autism in our sample. However, can we conclude anything about a relationship of this allele to SCZ or psychosis from our results?

Walss-Bass et al. [12] first genotyped 6 of 7 markers previously observed to be associated with SCZ in Iceland [13] in a sample of 134 trios with a history of psychosis including 94 cases diagnosed with SCZ, 16 cases with schizoaffective bipolar disorder, 4 cases with schizoaffective disorder depressed, 5 cases with major depressive episode accompanied by psychosis and 3 cases with psychosis not otherwise specified. They further stratified their sample into SCZ $(\mathrm{N}=114)$, manic psychosis $(\mathrm{N}=63)$, and non- manic psychosis cases. They did not find statistically significant evidence for over-transmission of alleles or haplotypes containing markers from the 'deCode' haplotype after correcting for multiple testing. However, they reported a trend ( $\mathrm{p}<0.05$ uncorrected for multiple testing) towards over transmission of the most common 4 marker haplotype that they observed in their sample $(\mathrm{f}=$ 0.168 ), to cases with manic psychosis. Similarly, alleles at the two microsatellite markers in this core haplotype were not significantly associated with manic psychosis after correction for multiple testing.

Walss-Bass et al. [2] subsequently selected the 12 individuals with a history of SCZ or psychosis and mania that carried alleles representing the most common haplotype observed in cases for sequencing. They found a $\mathrm{G}>\mathrm{T}$ polymorphism in exon 11 resulting in a Val>Leu missense change observed in 1 of the 12 cases. However, this exon 11 variant was not in LD with the haplotype that they used to guide their search for a disease polymorphism so it may represent a serendipidous finding. They next genotyped the exon 11 variant in 378 individuals from 151 different CVCR families, 142 of whom were affected with psychosis. The T allele was transmitted 13 times from heterozygous parents to affected individuals and not transmitted 4 times yielding a significant association with psychosis $(Z=2.810, \mathrm{p}=0.0049)$ and with $\operatorname{SCZ}(Z=2.383, \mathrm{p}=$ 
0.0191) using the Family-based Association Test (FBAT). The authors then found genealogical connections linking the families transmitting the T-allele to one common Spanish founder. Our data do show evidence of a possible founder effect surrounding the T-allele in 3 independent individuals (and possibly in another 2 independent individuals) however none of those families were segregating SCZ.

\section{Conclusion}

In summary, our data does not support a relationship of the NRG1 exon 11 missense variant with either autism or SCZ in the CVCR, although it does not reduce support for the previous association with SCZ either. Further studies examining the frequency of the exon $11 \mathrm{~T}$ allele in control individuals from the CVCR, and LD in the vicinity of the exon 11 variant should help determine whether the NRG1 allele actually does contribute to psychosis in this population.

\section{Abbreviations}

CVCR Central Valley of Costa Rica, NRG1 Neuregulin 1, schizophrenia SCZ.

\section{Competing interests}

The author(s) declare that they have no competing interests.

\section{Authors' contributions}

LAM analyzed the genotyping data and wrote the manuscript, LO and AN performed the microsatellite and Taqman genotyping and assisted with analysis of the genotyping data. PJG, ERM, ME, SMM and MFD performed the clinical evaluation of the probands and reviewed the case histories of all 'T-allele' carriers. All authors read and approved of the manuscript.

\section{Acknowledgements}

This work was supported by NINDS grant number R0I 043540, a Young Investigator Award from the Seaver Center for Excellence in Autism Research and a grant from the General Clinical Research Center at the Mount Sinai School of Medicine (all to LAM).

We thank Luz Ospina for her help editing and formatting this manuscript, Pamela Bondy for her help best-estimating cases and Safiana Katz for her careful processing of immortalized cell lines. We also thank Eduardo Fournier for genealogical analyses, Dr. Olga Arguedas from the Bioethical Board of the Hospital Nacional de Ninos and Dr. Abdon Castro, Presidente de la Fundación Pro Hospital Nacional de Ninos "Dr Carlos Sáenz Herrera."

Above all, we are grateful to the families who have participated in our study, and to the Autism Parents' Association of San José, Costa Rica.

\section{References}

I. McInnes LA, Gonzalez PJ, Manghi ER, Esquivel M, Monge S, Delgado $M F$, Fournier $E$, Bondy P, Castelle $K$ : A genetic study of autism in Costa Rica: multiple variables affecting IQ scores observed in a preliminary sample of autistic cases. BMC Psychiatry 2005, 5: 15.

2. Walss-Bass C, Liu W, Lew DF, Villegas R, Montero P, Dassori A, Leach RJ, Almasy L, Escamilla M, Raventos $\mathrm{H}$ : A novel missense mutation in the transmembrane domain of neuregulin $I$ is associated with schizophrenia. Biol Psychiatry 2006, 60:548-553.

3. Fatemi $\mathrm{SH}$ : Reelin glycoprotein in autism and schizophrenia. Int Rev Neurobiol 2005, 71:179-187.

4. Debbane M, Glaser B, David MK, Feinstein C, Eliez S: Psychotic symptoms in children and adolescents with $22 \mathrm{q} I \mathrm{I} .2$ deletion syndrome: Neuropsychological and behavioral implications. Schizophr Res 2006, 84:187-193.

5. Fine SE, Weissman A, Gerdes M, Pinto-Martin J, Zackai EH, McDonald-McGinn DM, Emanuel BS: Autism spectrum disorders and symptoms in children with molecularly confirmed $22 \mathrm{qII} .2$ deletion syndrome. J Autism Dev Disord 2005, 35:46I-470.

6. Rutter M, LeCouteur A, Lord C: Autism Diagnostic Interview Revised (ADI-R). Los Angeles: Western Psychological Services; 2003.

7. Lord C, Rutter M, DiLavore PC, Risi S: Autism Diagnostic Observation Schedule (ADOS). Los Angeles: Western Psychological Services; 2001.

8. Sparrow SS, Balla DA, Cicchetti DV: Vineland Adaptive Behavior Scales. UNITED STATES: American Guidance Service; 1984.

9. Larsson HJ, Eaton WW, Madsen KM, Vestergaard M, Olesen AV, Agerbo E, Schendel D, Thorsen P: Risk factors for autism: perinatal factors, parental psychiatric history, and socioeconomic status. Am J Epidemiol 2005, 161:916-25. discussion 926-8

10. Li T, Stefansson H, Gudfinnsson E, Cai G, Liu X, Murray RM, Steinthorsdottir V, Januel D, Gudnadottir VG, Petursson H, Ingason $\mathrm{H}$, Gulcher JR, Stefansson K, Collier DA: Identification of a novel neuregulin I at-risk haplotype in Han schizophrenia Chinese patients, but no association with the Icelandic/Scottish risk haplotype. Mol Psychiatry 2004, 9:698-704.

11. Walss-Bass C, Raventos H, Montero AP, Armas R, Dassori A, Contreras S, Liu W, Medina R, Levinson DF, Pereira M, Leach RJ, Almasy $L$, Escamilla MA: Association analyses of the neuregulin I gene with schizophrenia and manic psychosis in a Hispanic population. Acta Psychiatr Scand 2006, I I 3:3 |4-32I.

12. Stefansson H, Sigurdsson E, Steinthorsdottir V, Bjornsdottir S, Sigmundsson T, Ghosh S, Brynjolfsson J, Gunnarsdottir S, Ivarsson O, Chou TT, Hjaltason O, Birgisdottir B, Jonsson H, Gudnadottir VG, Gudmundsdottir E, Bjornsson A, Ingvarsson B, Ingason A, Sigfusson S, Hardardottir H, Harvey RP, Lai D, Zhou M, Brunner D, Mutel V, Gonzalo A, Lemke G, Sainz J, Johannesson G, Andresson T, Gudbjartsson D, Manolescu A, Frigge ML, Gurney ME, Kong A, Gulcher JR, Petursson $H$, Stefansson K: Neuregulin I and susceptibility to schizophrenia. Am J Hum Genet 2002, 7 I:877-892.

13. Therapeutic Pipeline [http://www.decode.com/nrgl/markers/]

\section{Pre-publication history}

The pre-publication history for this paper can be accessed here:

\section{http://www.biomedcentral.com/1471-244X/7/21/pre} pub

Publish with Bio Med Central and every scientist can read your work free of charge

"BioMed Central will be the most significant development for disseminating the results of biomedical research in our lifetime. "

Sir Paul Nurse, Cancer Research UK

Your research papers will be:

- available free of charge to the entire biomedical community

- peer reviewed and published immediately upon acceptance

- cited in PubMed and archived on PubMed Central

- yours - you keep the copyright

BiolMedcentral 